\title{
Cognitive Workload and Psychophysiological Parameters During Multitask Activity in Helicopter Pilots
}

Sophie Gaetan; Erick Dousset; Tanguy Marqueste; Lionel Bringoux; Christophe Bourdin; Jean-Louis Vercher; Patricia Besson

BACKGROUND: Helicopter pilots are involved in a complex multitask activity, implying overuse of cognitive resources, which may result in piloting task impairment or in decision-making failure. Studies usually investigate this phenomenon in well-controlled, poorly ecological situations by focusing on the correlation between physiological values and either cognitive workload or emotional state. This study aimed at jointly exploring workload induced by a realistic simulated helicopter flight mission and emotional state, as well as physiological markers.

METHOD: The experiment took place in the helicopter full flight dynamic simulator. Six participants had to fly on two missions. Workload level, skin conductance, RMS-EMG, and emotional state were assessed.

RESULTS: Joint analysis of psychological and physiological parameters associated with workload estimation revealed particular dynamics in each of three profiles. 1) Expert pilots showed a slight increase of measured physiological parameters associated with the increase in difficulty level. Workload estimates never reached the highest level and the emotional state for this profile only referred to positive emotions with low emotional intensity. 2) Non-Expert pilots showed increasing physiological values as the perceived workload increased. However, their emotional state referred to either positive or negative emotions, with a greater variability in emotional intensity. 3) Intermediate pilots were similar to Expert pilots regarding emotional states and similar to Non-Expert pilots regarding physiological patterns.

DIscussıon: Overall, high interindividual variability of these results highlight the complex link between physiological and psychological parameters with workload, and question whether physiology alone could predict a pilot's inability to make the right decision at the right time.

KEYWORDS: workload, physiological parameters, emotional state, multitask activity, helicopter pilots.

Gaetan S, Dousset E, Marqueste T, Bringoux L, Bourdin C, Vercher J-L, Besson P. Cognitive workload and psychophysiological parameters during multitask activity in helicopter pilots. Aerosp Med Hum Perform. 2015; 86(12):1052-1057.

$\mathrm{P}$ iloting a helicopter is a complex multitask activity involving highly demanding decisional processes which need to occur in a minimal amount of time. Thus, many subtasks compete for limited processing capacity. ${ }^{14}$ Piloting is part of extreme iterative practices, meaning that conditions may generate intense emotions and require substantial adaptation to the situation, potentially perceived by the participant as beyond his/her means. ${ }^{13}$ This adaptive process is related to cognitive workload, which corresponds to the cognitive resources mobilized for processing information, memorizing it, and making a decision. However, a too high level of cognitive workload can exceed resources and adaptive capacities, traditionally defined as a cognitive overload, ${ }^{9}$ corresponding to a mismatch between cognitive resources mobilized and available and cognitive resources required by the task. To reduce the risk of overload, efficient aiding systems should be able to assess the psychophysiological state of pilots to help them to allocate cognitive resources adapted to the current situation. ${ }^{11}$

From Aix-Marseille Université, CNRS, Institut des Sciences du Mouvement: Etienne-Jules Marey, Marseille, France.

This manuscript was received for review in December 2014. It was accepted for publication in September 2015.

Address correspondence to: Sophie Gaetan, Faculté des Sciences du Sport, 163 avenue de Luminy, Case 910, 13288 Marseille Cedex 09, France; sogaetan@gmail.com.

Reprint \& Copyright ( $\odot$ by the Aerospace Medical Association, Alexandria, VA.

DOI: $10.3357 /$ AMHP.4228.2015 
The potential use of physiological measurements to determine cognitive workload and emotional state was poorly explored in the specific context of helicopter piloting. ${ }^{2}$ Ang et al. stressed the difficulty of estimating psychological and cognitive states based on physiological measurements, since variations in physiological signals are primarily related to changes in pilots' physical state. ${ }^{1}$ Therefore, all these measurements appear to be sensitive to huge variations in psychophysiological states without being specific to particular psychophysiological states. Thus, using a single physiological marker cannot be sufficient to characterize cognitive workload and the emotional state of a pilot.

Several authors suggested that a combination of several physiological markers could be used as an efficient indicator of cognitive workload and emotional state of the pilot, ${ }^{4,16}$ and recommended mission flight analysis combined with physiological measurement. ${ }^{5}$ However, Philip et al. ${ }^{12}$ established that those predictive models made in-lab are not transferable to more ecologically realistic situations. The latter enhance individual variability expression. Indeed, emotional state may affect the adaptive capacity of the participants, and thus their workload, and may also influence decision-making. ${ }^{7}$ Similarly, workload and emotions induce neurovegetative and neuroendocrine interactions, resulting in sudden changes in physiological markers. ${ }^{10}$

Correlation between psychology, physiology, and workload has been poorly explored so far in the helicopter pilot. To the best of our knowledge, no specific indicator linking workload to emotional state could be brought out. Thus, describing the relationships between emotional, physiological, and psychological states and workload would represent a promising way to improve aiding systems. Since we wanted to focus on a better understanding of interindividual differences, we analyzed physiological markers jointly with emotional states on a subjectby-subject basis to extract useful information about cognitive workload. Thus, the purpose of our study was to investigate the way physiological and psychological markers may refer to individual levels of workload using the paradigm of helicopter piloting in a ultra-realistic dynamic simulator used to train experienced pilots to a new model of helicopter.

\section{METHODS}

\section{Subjects}

Six trained pilots capable of handling flight with a 10-ton class helicopter simulator were selected and informed about the study, its design, and the advantages/disadvantages of participation. The study protocol was approved in advance by AixMarseille Université Ethical Committee in accordance with the declaration of Helsinki. Each subject provided written informed consent before participating.

\section{Procedure}

The experiment took place in the helicopter full flight dynamic simulator at the Helisim Corporation (Marignane, France). The participants had to fly on two offshore missions, taking off from Marseille Provence airport and reaching in turn different offshore platforms. Each mission lasted up to $30 \mathrm{~min}$ separated by 15 min. Mission 1 (M1) was the reference mission; mission 2 (M2) involved facing extreme situations (cockpit and/or system and/or engine failure, critically low fuel level, stormy weather...) requiring high cognitive resources and adaptation capacities. For each mission, scenarios were defined so as to induce different degrees of difficulty and workload levels through the addition of different incidents.

During the simulations, participants were asked to verbally rate their workload level every 1.5 min using a rating scale from 1 (very low) to 4 (very high). Then, for each participant and for each mission, we calculated the time spent at each level of workload. These segments were expressed as a percentage of the total duration of the mission.

Before the experiment, participants remained quiet in a dark room in order to record basal values of skin conductance (SC). The latter was assessed using two electrodes positioned at the extremities of the right index and auricular fingers. Root mean square of the electromyogram (RMS-EMG) of the right common finger flexor (CFF) and both descendent trapezius muscles (DT) were calibrated with respect to their minimal and maximal values. All the signals mentioned hereafter were acquired according to SENIAM recommendations. ${ }^{18}$ Signal synchronization was done using Captiv Software (TEA, Paris, France).

Emotional states were evaluated using the Izard differential emotions scale ${ }^{8}$ divided into 3 dimensions: positive (score range from 0 to 24); neutral (from 0 to 12); and negative (from 0 to 84 ). We evaluated emotional state before the flight (Time 1), after M1 (Time 2), and at the end of M2 (Time 3).

\section{RESULTS}

We aimed to better understand interindividual responsiveness of physiological markers with respect to psychological individual characteristics. Thus, we could not perform a classical statistical analysis, but rather looked at specific patterns for each participant in light of his personal profile. In line with this purpose, we successively present workload, and physiological and psychological results separately for each participant (Table I, Fig. 1). We rather consider as strength and as proof of robustness that results are as close as possible to those which could be recorded in flight during real operations.

Participant 1 (P1) remained mostly at the lowest workload level during $73 \%$ of M1. He spent the rest of the mission at level 2. During M2, P1 showed the lowest workload level during $18 \%$ of the mission. He remained mostly at a low workload level, reaching level 3 during only $35 \%$ of the time. This participant never reached the highest workload level. SC tended to exhibit a slight decrease when perceived workload increased (Fig. 2). RMS-EMG values increased with the perceived difficulty of the task (Fig. 3). However, RMS-EMG changes remained at a low level, particularly for both trapezes muscles. P1 only felt positive emotions, with a moderate intensity, which tended to decrease. 
Table I. Emotional State (Negative, Neutral, and Positive Dimensions) According to the Three Measurement Times (from Time 1 to Time 3) for Each Participant (from P1 to P6).

\begin{tabular}{|c|c|c|c|c|c|c|c|c|c|}
\hline & \multicolumn{3}{|c|}{ NEGATIVE } & \multicolumn{3}{|c|}{ NEUTRAL } & \multicolumn{3}{|c|}{ POSITIVE } \\
\hline & TIME 1 & TIME 2 & TIME 3 & TIME 1 & TIME 2 & TIME 3 & TIME 1 & TIME 2 & TIME 3 \\
\hline P1 & 0 & 0 & 0 & 0 & 0 & 0 & 15 & 13 & 12 \\
\hline P2 & 0 & 0 & 0 & 0 & 0 & 0 & 10 & 8 & 5 \\
\hline P3 & 1 & 1 & 1 & 2 & 2 & 1 & 12 & 11 & 12 \\
\hline P4 & 0 & 1 & 1 & 0 & 1 & 4 & 22 & 22 & 21 \\
\hline P5 & 8 & 5 & 11 & 5 & 7 & 7 & 18 & 18 & 15 \\
\hline P6 & 2 & 2 & 3 & 0 & 0 & 1 & 17 & 17 & 17 \\
\hline
\end{tabular}

As for P1, participant $2(\mathrm{P} 2)$ remained mostly at the lowest workload level during $54 \%$ of $\mathrm{M} 1$ and he spent the rest of the mission at level 2. During M2, this participant also returned to the lowest workload level during $19 \%$ of time. Like P1, he remained mostly at a low workload level and he reached level 3 during $40 \%$ of time. The highest workload level was never reached. SC values remained stable with workload level. Similarly to P1, P2 RMS-EMG values increased with the perceived difficulty of the task. However, absolute values of RMS-EMG remained at a low level. $\mathrm{P} 2$ felt only positive emotions, like P1, with a low intensity (from 5 to 10 ), which tended to decrease.

Participant 3 (P3) remained at the lowest workload level during $38 \%$ of M1. He remained mostly at level 2. During M2, P3 was not able to return to level 1 . He remained at level 2 during $13 \%$ of the mission and remained mostly at level 3 during $53 \%$ of the mission. He reached the highest workload level during 34\% of the mission. SC in P3 decreased with the increase in perceived workload. CFF RMS-EMG had a tendency to increase from levels 1 to 3 and decreased from levels 3 to 4 . DT RMSEMG remained at very low level with a decrease from level 2 to 4. P3 felt positive emotions at a stable and moderate intensity. He felt neutral and negative emotions at a stable and very low intensity.

Participant 4 (P4) remained at the lowest workload level during $20.9 \%$ of M1. Like P3, P4 remained mostly at level 2. Then he reached level 3 during $8.6 \%$ of the time. During M2, P4 was, like P3, not able to return to the minimum workload level.
He reached level 2 during 22.8\% of the mission and level 3 during $33.7 \%$ of the time. $\mathrm{P} 4$ remained mostly at the highest workload level during $43.5 \%$ of the mission. P4, as P3, exhibited multiple variations. Indeed, SC appeared to greatly oscillate. CFF RMSEMG increased from level 1 to 3 and then, as with P3, decreased from levels 3 to 4 . Right DT RMS-EMG slightly decreased from level 1 to 4 while left DT RMS-EMG remained at a very low level, indicating a possible disengagement of this particular muscle. $\mathrm{P} 4$ felt positive emotions at a stable but high intensity. He felt neutral emotions at a low intensity, which tended to increase at Time 3. Like P3, he felt negative emotions at a stable and very low intensity.

Participant 5 (P5) remained at the lowest workload level during $28 \%$ of M1. Like P3 and P4, P5 remained mostly at a level 2. Then, like P4, he reached level 3 during 19\% of the time. During M2, P5 returned to the lowest workload level during $15.8 \%$ of the mission. He remained mostly at level 2 during $45.6 \%$ of the time. He reached level 3 during a third of the time and the highest workload level during $9 \%$ of the mission. Sometimes this participant did not respond to the verbal workload level request. P5 exhibited a profile similar to P4. Indeed, SC decreased from levels 1 to 3 and then increased from levels 3 to 4. CFF RMS-EMG increased from level 1 to 3 and then decreased from levels 3 to 4 . Right DT RMS-EMG decreased from level 1 to 3 and then slightly increased from levels 3 to 4 . Left DT RMS EMG remained at a very low level, as for P4. P5 felt positive emotions with a high intensity, but these emotions tended to decrease at Time 3. He felt neutral and negative emotion at a variable and moderate intensity. The neutral dimension tended to increase at Time 2 and the negative dimension tended to decrease at Time 2 and to increase at Time 3.

Participant 6 (P6) remained at the lowest workload level during 35.5\% of M1. Like P3, P4, and P5, P6 remained mostly
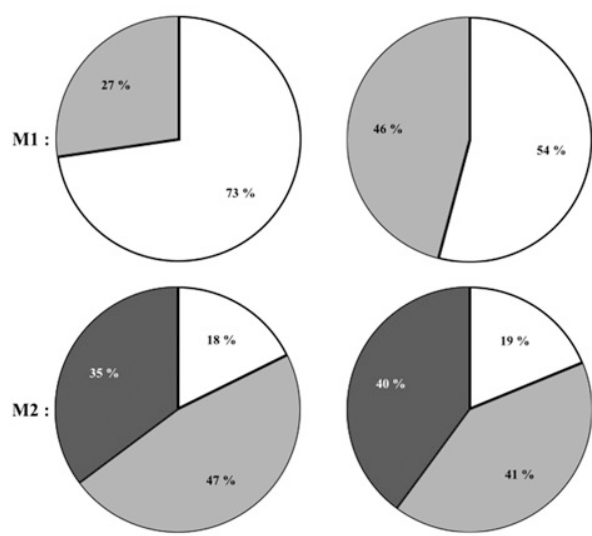

S1

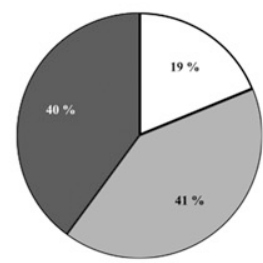

S2

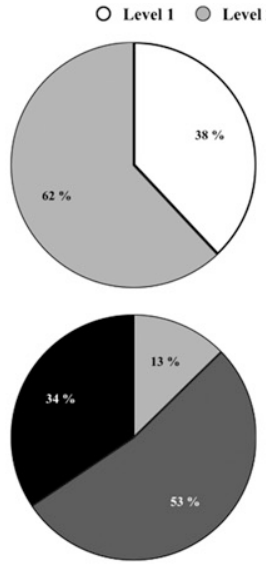

S3
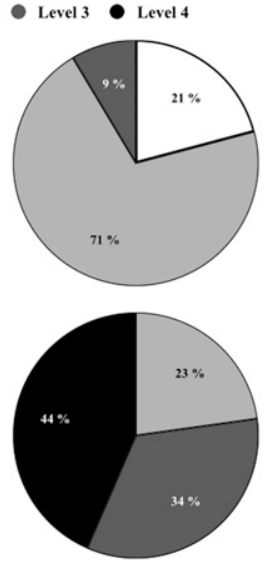

S4
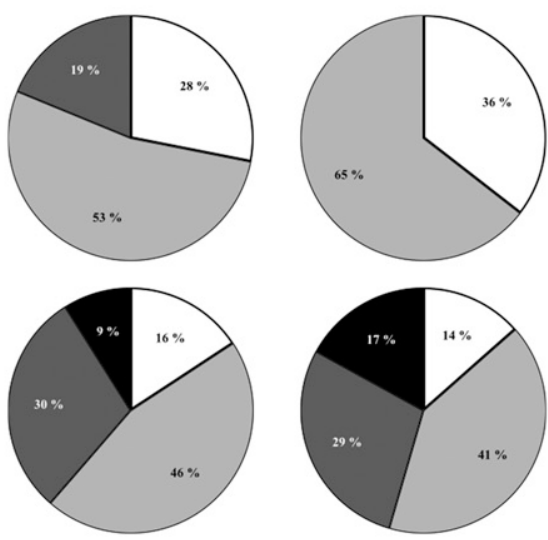

s5

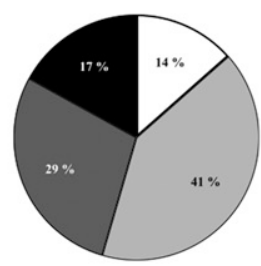

S6

Fig. 1. Workload changes for each participant (from P1 to P6; labeled as S1 to S6 in the figure). Each graph indicates time spent (\% total time) at each self-assessed workload (from Level 1 to Level 4) for each mission; M1 and M2 are Mission 1 and Mission 2. 
$\square$ Level $1 \square$ Level $2 \square$ Level $3 \square$ Level 4

Skin conductance, $\%$ basal values

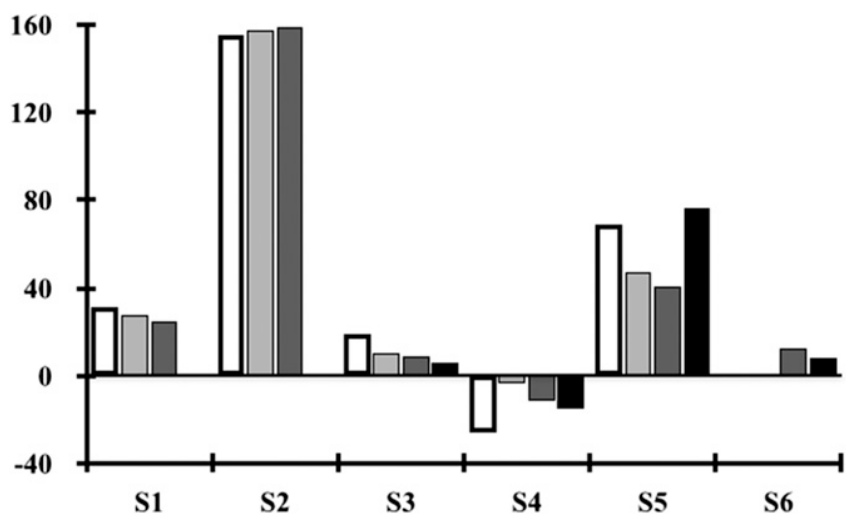

Fig. 2. Skin conductance results. Related to level of workload, changes in skin conductance are expressed as percentage of the baseline values.

at level 2. During M2, like P1, P2, and P5, P6 returned to the lowest workload level during $13.5 \%$ of the mission and he remained mostly at level 2 . He reached level 3 during $28.5 \%$ of time. He reached the highest workload level during $17 \%$ of the mission. Sometimes, like P5, this participant did not respond to the verbal workload level request. His SC increased from levels 1 to 3 and then decreased from levels 3 to 4 . CFF RMS-EMG increased from perceived workload levels 1 to 4 . Right DT RMS-EMG exhibited a profile similar to CFF. Left DT RMSEMG remained at a very low level, but remained stable. Like P3 and P4, P6 felt positive emotions at a stable and high intensity, and neutral and negative emotions at a stable and very low intensity.

\section{DISCUSSION}

Many systems have been designed in order to prevent cognitive overload during real flights, either by helping the decisionmaking process or by automatizing tasks. However, these processes can be sources of risk by themselves, resulting in cognitive overload or in a lack of vigilance, which could be considered an emotional risk of misbehavior. ${ }^{15}$ Secondly, these systems are mainly generic despite the fact that interindividual differences may exist at the physiological and psychological levels. For these reasons, this study aimed at individually exploring the evolution of workload induced by the multitask activity required in helicopter piloting in relation to emotional state and physiological markers. In our experiment, combined analysis of physiological and psychological markers related to workload provided a more precise analysis of helicopter pilots' adaptive abilities during missions.

Low changes in physiological data combined with positive emotions felt at low intensity and at a low level of perceived workload suggest that P1 and P2 were able to adjust their motor behavior to the level of difficulty, keeping self-control and capacity to handle stress induced by the task, hence making appropriate decisions. These psychological and physiological considerations establish that these two participants remained analytical and were not, or very little, emotionally affected during the flight. Indeed, P1 exhibited a cold but precise analysis of the situation whatever the difficulty and was never overwhelmed or in a state of difficulty. He remained calm and always selected the adapted solution to the different problems. P2 exhibited a behavior similar to P1. In addition, he always justified his decisions and his workload self-evaluations, demonstrating his ability to take a global view of situations.

Physiological and psychological data suggest that $\mathrm{P} 3$ and $\mathrm{P} 4$ were overwhelmed by high levels of difficulty and were unable to continue the mission. It is not surprising since flying a helicopter requires great coordination due to the simultaneous application of forces to the cyclic, collective, and pedal controls. ${ }^{3}$ Consequently, we can consider that P3 and P4 felt more than they analyzed, contrary to P1 and P2. Indeed, during the first mission, P3 crashed the helicopter several times before successfully landing. During the second mission, distance between the helicopter and the platform pylon was underestimated, resulting in collision. He was unable to focus on all details and did not detect a low level of fuel. Despite apparent
Fig. 3. RMS-EMG results from A) the common flexor digitorum, B) the right trapezius descendens, and C) the left trapezius descendens. Related to level of workload, changes in RMS-EMG are expressed as percentage of maximum voluntary force. 
relaxation and happiness, $\mathrm{P} 4$ reported a high level of fatigue. $\mathrm{He}$ did not succeed in analyzing multiple sources of information during the task, resulting in an inability to detect failures such as low level of fuel or engine failures. Another manifestation of this inability was erratic flight.

It is noteworthy to indicate that physiological analyses confirmed P5 and P6 status as similar to P3 and P4 while psychological analysis suggested that P6 followed the profile of P1 and P2, contrary to P5. Interestingly, contrast of the psychological to physiological analyses suggested that P5 and P6 did not have the adaptive capability to manage a situation and the associated workload with information flow. Clearly, these pilots were able to face and handle the flight until a given threshold of workload was reached. Indeed, the higher the cognitive effort, the lower the performance and the higher the risk for the pilot. Moreover, when the cognitive cost required by the task became too high and exceeded the participant's capacity, "cognitive presence" seemed to disappear and the participant "let go." In other words, the participant might become overwhelmed by the task or the situation and no longer be able to control or organize his actions in order to adapt and face the situation. This is confirmed by the observed behavior of these two participants. P5 explained that he was able to analyze security problems by compartmentalizing and prioritizing the different information. However, as the difficulty increased, he could no longer cope with the situation and was overwhelmed. As a consequence, he showed difficulties in performing and managing multiple tasks and information that resulted in a crash. P6, despite a lack of knowledge about this 10-ton class helicopter specificity, took off and flew successfully, showing his piloting abilities. He successfully managed different information as far as low levels of difficulty. With the increase of difficulty and failures, he became unable to handle simultaneous visual and instrumental flight. At the end of the second mission, he was overwhelmed and he did not realize that he rotated several times around the landing target. At the end of the experiment, he expressed total exhaustion. Taken together, P5 and P6 had knowledge and some expertise in piloting, so that they at first seemed able to handle the mission. However, they were quickly overwhelmed by the task difficulty.

Noticeably, our scenarios were specifically designed to induce the highest levels of workload, including rare and dramatic events, probably leading to overwhelming situations. Overall, our results suggest that high variability of physiological values is associated to more emotional than analytical management of a situation. Consequently, a risk of overload and of wrong decision making during a flight mission could occur. Complementarily, when slight changes in physiological values were concomitant to slight changes in emotional state, these pilots remained analytical all through the flight. Even if these results should be explored in a larger population to be refined, they have the merit to question through physiological measurements whether pilots are able or not to make the right decision at the right time. Even if helicopter pilots have to handle multitasking activity in a limited amount of time, the question still remains as to whether the impairment of pilots psychophysiological state reduces piloting performance or not. ${ }^{17}$ Indeed, according to Sarter et al., ${ }^{15}$ a pilot could have inappropriate reactions following blindly automated procedures displayed on their multifunction screens because of a lack of analysis of the situation and of a too high a level of faith in the automated process. Further studies will be necessary to assess the level of changes in physiological markers which characterizes a nonoptimal emotional state for the pilot to handle his/her workload.

In summary, individualized joint analysis of psychological parameters and physiological parameters associated with workload estimates reveals particular dynamics, allowing us to describe three profiles. 1) Expert pilots who demonstrated a slight increase of measured physiological parameters associated with the increase of difficulty level. Workload estimates, however, never reached the highest level and emotional state for this profile only referred to positive emotions, with a low emotional intensity. 2) Non-Expert pilots demonstrated increasing physiological values as the perceived workload increased. However, their emotional state referred to both positive and negative emotions, with a greater variability in emotional intensity. 3) Intermediate pilots were close to Expert pilots regarding emotional states and close to Non-Expert pilots regarding physiological patterns. Overall, these findings emphasize the impossibility of establishing an accurate prediction of cognitive workload without taking into account individual specificities and crossed analyses of multiple psychophysiological data. In line with these observations, it appears that the development of a generic crew assistant for helicopter pilots, based on a few physiological or psychological measures, remains rather a utopia at this stage.

\section{ACKNOWLEDGMENTS}

The authors gratefully acknowledge Airbus Helicopters for their support and for the opportunity to access the dynamic flight simulator. They also acknowledge M. Gillet as well as the Helisim's staff for their help in defining and running the simulator experiment. Finally, many thanks to Pr. Pierre Therme, who closely collaborated with the project, and to all the participants (pilots) who took part in the experiment.

Authors and affiliations: Sophie Gaetan, M.Sc., Ph.D., Erick Dousset, Ph.D., Tanguy Marqueste, M.Sc., Ph.D., Lionel Bringoux, M.Sc., Ph.D., Christophe Bourdin, Ph.D., Jean-Louse Vercher, Ph.D., Patricia Besson, M.Sc., Ph.D., AixMarseille Universite, CNRS, Institut des Sciences du Mouvement: Etienne-Jules Marey, Marseille, France.

\section{REFERENCES}

1. Ang B, Linder J, Harms-Ringdahl K. Neck strength and myoelectric fatigue in fighter and helicopter pilots with a history of neck pain. Aviat Space Environ Med. 2005; 76(4):375-380.

2. Besson P, Bourdin C, Bringoux L, Dousset E, Maiano C, et al. Effectiveness of physiological and psychological features to estimate helicopter pilots' workload: a Bayesian network approach. IEEE Transactions on Intelligent Transportation Systems. 2013; 14(4):1872-1881. 
3. Billings CE, Eggspuehler JJ, Gerke RJ, Chase RC. Studies of pilot performance: II. Evaluation of performance during low altitude flight in helicopters. Aerosp Med. 1968; 39:19-31.

4. Cain B. Review of the mental workload literature. In: Development DRa, ed. Report \#RTO-TRHFM-121-Part-II. Toronto, Ontario Canada: Defence Research and Development Canada-Toronto, Human System Integration Section; 2007.

5. Haarmann A, Boucsein W, Schaefer F. Combining electrodermal responses and cardiovascular measures for probing adaptive automation during simulated flight. Appl Ergon. 2009; 40(6):1026-1040.

6. Hoc JM, Amalberti R. Cognitive control dynamics for reaching a satisfying performance in complex dynamic situations. J Cogn Eng Decis Mak. 2007; 1:22-55.

7. Isen AM. An influence of positive affect on decision making in complex situations: Theoretical issues with practical implications. J Consum Psychol. 2001; 11(2):75-85.

8. Izard CE. Human emotions. New York: Plenum Press; 1977.

9. Karasek RA, Theorell T. Healthy work. Stress, productivity and the reconstruction of working life. New York: Basic Books; 1990.

10. Lehrer P, Karavidas M, Lu SE, Vaschillo E, Vaschillo B, Cheng A. Cardiac data increase association between self-report and both expert ratings of task load and task performance in flight simulator tasks: An exploratory study. Int J Psychophysiol. 2010; 76(2):80-87.

11. Norcio AF, Stanley J. Adaptive human-computer interfaces: a literature survey and perspective. IEEE Transactions on Systems, Man and Cybernetics. 1989; 19(2):399-408.

12. Philip P, Sagaspe P, Taillard J, Valtat C, Moore N, et al. Fatigue, sleepiness, and performance in simulated versus real driving conditions. Sleep. 2005; 28(12):1511-1516.

13. Rivolier J. Stress et situations extrêmes. Paris (France): Presses Universitaires de France; 1995.

14. Roscoe A. Aviation psychology. Ames (IA): Iowa State University Press; 1980.

15. Sarter NB, Mumaw RJ, Wickens CD. Pilots' monitoring strategies and performance on automated flight decks: an empirical study combining behavioral and eye-tracking data. Hum Factors. 2007; 49(3):347-357.

16. Sequeira H, Hot P, Silvert L, Delplanque S. Electrical autonomic correlates of emotion. Int J Psychophysiol. 2009; 71(1):50-56.

17. Szafran J. Psychological studies of aging in pilots. Aerosp Med. 1969; 40(5):543-553.

18. SENIAM. European recommendations for Surface Electromyography. Enschede (The Netherlands): SENIAM Project Management Office; 1999.
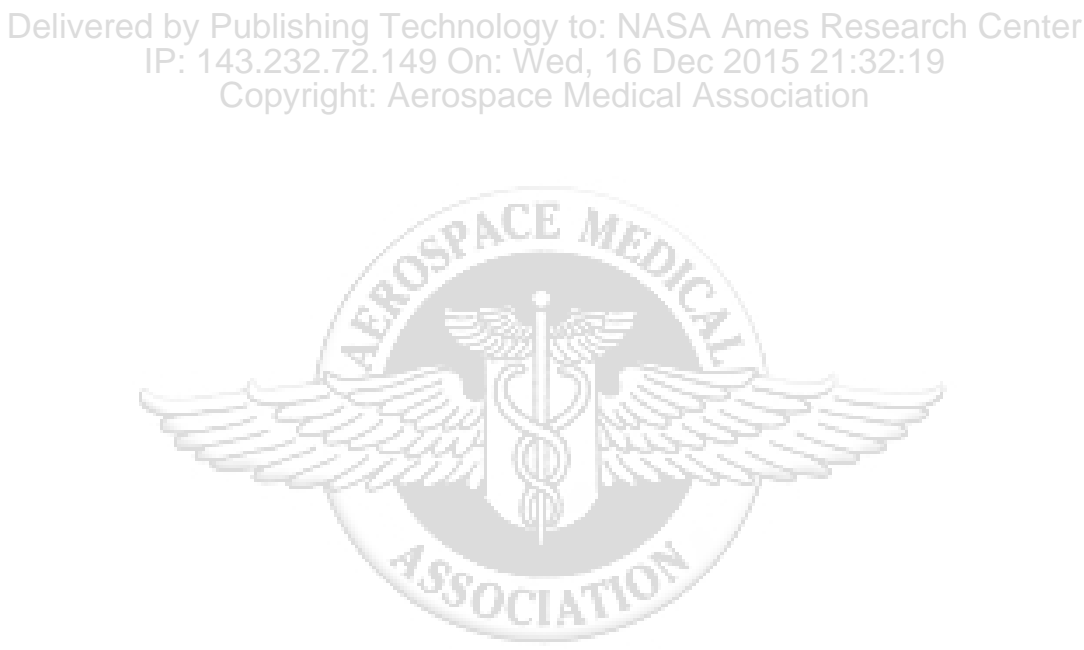